\title{
Energy Conservation and Gravitational Wavelength Effect of the Gravitational Propagation Delay Analysis
}

\section{Philipp Kornreich}

1090 Wien, Austria

King of Prussia, PA 19406, USA

Email: pkornrei@syr.edu

How to cite this paper: Kornreich, P. (2017) Energy Conservation and Gravitational Wavelength Effect of the Gravitational Propagation Delay Analysis. Journal of Applied Mathematics and Physics, 5, 912921.

https://doi.org/10.4236/jamp.2017.54080

Received: February 14, 2017

Accepted: April 27, 2017

Published: April 30, 2017

Copyright $\odot 2017$ by author and Scientific Research Publishing Inc. This work is licensed under the Creative Commons Attribution International License (CC BY 4.0).

http://creativecommons.org/licenses/by/4.0/

\begin{abstract}
The motion of objects where the interaction propagated with a finite velocity was analyzed in my previous paper "The Contribution of the Gravitational Propagation Delay to Orbital and Center of Mass Motions". It is shown here that this analysis is valid for the case when the wavelength of the gravitational wave excited by the motion of the masses is much larger than the system of masses. It is also proven here that the conclusion reached in my previous paper conserves energy. Since this interaction is conservative, the energy is equal to the Hamiltonian. Therefore, the Hamiltonian is calculated and it is shown that the time derivative of the Hamiltonian is equal to zero. Thus, the Hamiltonian and therefore, the energy, are constants.
\end{abstract}

\section{Keywords}

Propagation Delay, Gravitation, Newtonian Mechanics, Lagrangian, Hamiltonian, Constants, Gravitational Waves

\section{Introduction}

It is determined here, that for the case when the wavelength of the gravitational wave generated by the motion of the point objects are much larger than the distance between objects, Newtonian Classical Mechanics gives accurate results. The correction to Classical Mechanics due to the curvature of space-time in this limit, is approximately the same size as the ratio of the Schwarzschild radius divided by the distance between objects. The curvature of space-time due to the masses is described by the General Relativity Theory. An equation for the wavelength of the gravitational wave generated by the motion of the objects is derived. 
The motion of objects where the interaction propagated with a finite velocity was analyzed in my previous paper "The Contribution of the Gravitational Propagation Delay to Orbital and Center of Mass Motions" [1]. This analysis has been questioned if the result conserves energy. Here it is proven that the model in the above paper does conserve energy.

\section{Comparison of Gravitational Wavelength and System Size}

The gravitational interaction is modeled by the General Relativity Theory as a deformation of space-time caused by the presence of masses [2]. Mathematically, the space-time continuum is described by the geodetic tensor. The deformation of the space-time continuum by moving masses propagates as Gravitational Waves is schematically shown in Figure 1. Indeed, Einstein's equations have wave like solutions for space-time. Gravitational Waves have recently been measured [3].

The gravitational interaction propagates with the speed of light among objects. The obvious way to model this interaction is by the gravitational waves derived by the General Relativity Theory. However, for the objects considered in this paper, the gravitational waves generated by the motion of these objects have wavelengths very much larger than the size of the system of the objects, see Figure 1. Indeed, the reason that the Newtonian model is so successful is that the gravitational waves generated by systems described by the Newtonian Classical Mechanics [4] have wavelengths substantially longer than the size of the systems.

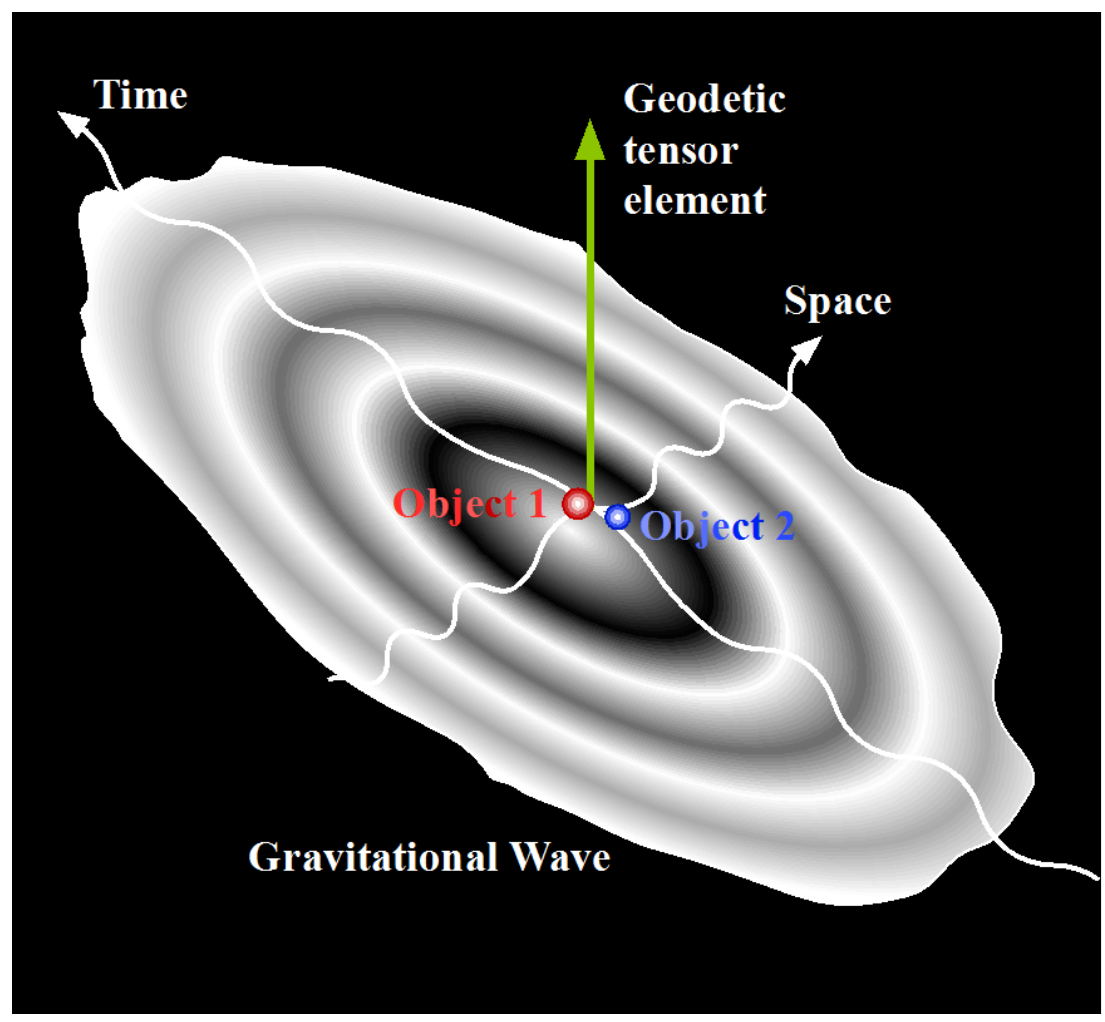

Figure 1. Schematic representation of a system with two objects in a deformed space-time continuum. The system consisting of Objects 1 and 2 is small compared to the wavelength of its gravitational wave. The coordinate axes follow the path of light beams. 
The oscillating and orbiting objects will cause steady state gravitational waves in the space-time continuum surrounding the objects. At large distances from the objects, the steady state gravitational waves are spherical-wave like. An expression for the wavelengths of the gravitational waves generated by the systems is derived in Appendix B. The calculations in Appendix B are based on a derivation in Appendix A. The gravitational waves travel with the speed of light. The wavelength $\lambda$ of this wave is derived in equation B $14 \mathrm{~b}$ of Appendix B and the equation below:

$$
\lambda=2 \pi R \sqrt{\frac{2 R}{r_{s s}}}
$$

where $R$ is the average distance between objects and $r_{s s}$ is the Schwarzschild radius of the sum of the masses of the objects. The Schwarzschild radius is given by:

$$
r_{\text {ss }}=\frac{2\left(m_{\text {Object } 1}+M_{\text {Object } 2}\right) G}{c^{2}} .
$$

The first result we have been seeking is given by Equation (1). For systems where the average distance $R$ between objects is much larger than the Schwarzschild Radius $r_{s s}$, the wavelength $\lambda$ of the Gravitational Wave is much larger than the system that generates the wave. For such systems, Newton's Classical Mechanics and the method to describe delayed interactions are very good mathematical models of nature.

For example, for the Earth Sun system $R$ is approximately equal to twice the orbital semi major axis. The semi major axis is equal to one astronomical unit $\mathrm{AU}=149,597,870,700 \mathrm{~m}$. The solar Mass $M_{\odot}$ is equal to $1.989 \times 1030 \mathrm{~kg}$. The mass of the Earth ME is equal to $5.972 \times 1024 \mathrm{~kg}$. This results in a Schwarzschild radius $r_{s s}$ which equals $2954.038 \mathrm{~m}$. For the Earth Sun system the wavelength of the gravitational wave is 89426.148 times longer than the average orbital diameter. Thus, the size of the Earth Sun system is equal to a very small fraction of the wavelength of the steady state gravitational wave it excites.

To determine if one could use a Newtonian approximation to calculate the measured gravitational waves resulting from the two colliding galaxies $R$ would have to be the distance between points in the galaxies, not the $410 \mathrm{Mpc}$ distance to us. Also, for the observation of the deflection of light from a star by the solar mass the static form of the deformed space-time continuum through which the light passes would have to be calculated. By using the General Relativity Theory one obtains that liht is deflected by the Solar mass. It is deflected by an angle of 4.24612 micro-radians, approximately equal to the Schwarzschild radius $r_{\mathrm{ss} \odot}$ of the Sun divided by the Sun radius $R_{\odot}$.

\section{Conservation of the Energy Analysis}

The Wavelength of the Gravitational Wave generated by the system is substantially larger than the size of the system analyzed here. Since this interaction is conservative, the energy is equal to the Hamiltonian. First, the Hamiltonian is 
calculated. Next, it is shown that the time derivative of the Hamiltonian is equal to zero. Thus, the Hamiltonian and therefore the energy are constants of the motion.

Here the initial steps of the propagation delay paper of reference [1] are restated:

The motion of the objects with a delayed interaction can be derived from a method similar to the Euler Lagrange model of Classical Mechanics [4]. One can develop a causal Lagrangian $L_{k}$ that contains the effect of the delayed gravitational interaction. Since the propagation time of the gravitational interaction is very short compared to the orbital period, one can extend the Lagrangian $L$ of the centrally symmetric Kepler problem [4] to include the propagation delay effect.

$$
\begin{gathered}
L=\frac{1}{2} m \dot{x}_{\mu} \dot{x}_{\mu}+\frac{1}{2} M \dot{y}_{\mu} \dot{y}_{\mu}+\frac{m M G}{\sqrt{\left(x_{\mu}-y_{\mu}\right)\left(x_{\mu}-y_{\mu}\right)}} \\
\rightarrow L_{k}=\frac{1}{2} m \dot{x}_{\mu k} \dot{x}_{\mu k}+\frac{1}{2} M \dot{y}_{\mu k} \dot{y}_{\mu k}+\frac{m M G}{2 \sqrt{\left(x_{\mu k}-y_{\mu k-2}\right)\left(x_{\mu k}-y_{\mu k-2}\right)}} \\
+\frac{m M G}{2 \sqrt{\left(y_{\mu k}-x_{\mu k-1}\right)\left(y_{\mu k}-x_{\mu k-1}\right)}}
\end{gathered}
$$

where summation over repeated Greek indices is implied. The Latin subscripts label discrete times. The single gravitational potential of the Kepler formulation is split into two potentials as shown in Equation (3). The first potential describes a gravitational interaction that was radiated by the Planet in the past at time $t_{k-2}$ and is sensed currently at time $t_{k}$ by the Moon. The second potential describes a gravitational interaction that was radiated by the Moon in the past at time $t_{k-1}$ and is sensed currently at time $t_{k}$ by the Planet. Thus, the Lagrangian $L_{k}$ is causal. Half of each of these potentials as described by Dirac [5] is used.

An equation of motion similar to the Euler Lagrange equation of motion is derived by a least action method described in my paper "Reaction Mechanics for Point Objects" [6]. The Euler Lagrange method for the derivation of the equations of motion is most readily implemented using tensor notation. Here $m$ is the mass and $x_{\mu k}$ is a component of the position vector $\boldsymbol{x}_{k}$ of the Moon at time step $t_{k}$ and $M$ is the mass and $y_{\mu k}$ is a component of the position vector $y_{k}$ of the Planet at time step $t_{k}$. The time differences such as $t_{k}-t_{k-1}$ are propagation delays. The $t_{k}-t_{k-1}$ are finite time durations. The time steps such as $t_{k-2}, t_{k-1}, t_{k}, t_{k+1}$, and $t_{k+2}$ are not necessarily consecutive. They are just time step labels. The time steps are not integer related. Bold letters such as $\boldsymbol{x}$ denote vectors, single superior dots such as $\dot{x}=\frac{\mathrm{d} x}{\mathrm{~d} t}$ denote time derivatives and double superior dots such as $\ddot{x}=\frac{\mathrm{d}^{2} x}{\mathrm{~d} t^{2}}$ denote second time derivatives.

The derivation of the Hamiltonian from the Lagrangian of Equation (3) is performed in Appendix A. The Hamiltonian Sum is the Legender transform [7] of the Sum of Lagrangians with respect to the current velocities for systems with delayed interactions. This calculation is performed in Appendix A. The time de- 
rivative of the Hamiltonian Sum with respect to a current time $t_{k}$ is given by Equation (A8) in Appendix A. Equation (A8) in the more compact vector form is rewritten below.

$$
\begin{aligned}
\frac{\mathrm{d} H}{\mathrm{~d} t_{k}}= & m \ddot{\boldsymbol{u}}_{1 k} \cdot \dot{\boldsymbol{u}}_{1 k}+\frac{m M G\left(\boldsymbol{u}_{1 k}-\boldsymbol{u}_{2 k-2}\right) \cdot \dot{u}_{1 k}}{\left|\boldsymbol{u}_{1 k}-\boldsymbol{u}_{2 k-2}\right|^{3}}+\frac{m M G\left(\boldsymbol{u}_{2 k}-\boldsymbol{u}_{1 k+1}\right) \cdot \dot{\boldsymbol{u}}_{1 k}}{\left|\boldsymbol{u}_{2 k}-\boldsymbol{u}_{1 k+1}\right|^{3}} \\
& +M \ddot{\boldsymbol{u}}_{2 k} \cdot \dot{\boldsymbol{u}}_{2 k}+\frac{m M G\left(\boldsymbol{u}_{2 k}-\boldsymbol{u}_{1 k-1}\right) \cdot \dot{u}_{2 k}}{\left|\boldsymbol{u}_{2 k}-\boldsymbol{u}_{1 k-1}\right|^{3}}+\frac{m M G\left(\boldsymbol{u}_{1 k}-\boldsymbol{u}_{2 k+2}\right) \cdot \dot{u}_{2 k}}{\left|\boldsymbol{u}_{1 k}-\boldsymbol{u}_{2 k+2}\right|^{3}}
\end{aligned}
$$

The equations of motion of the Moon and Earth are given by Equations (A3). Equations (A3) are rewritten here in the more compact form in vector notation.

$$
\begin{aligned}
& \text { а) } m \ddot{\boldsymbol{u}}_{1 k}+\frac{m M G\left(\boldsymbol{u}_{1 k}-\boldsymbol{u}_{2 k-2}\right)}{\left|\boldsymbol{u}_{1 k}-\boldsymbol{u}_{2 k-2}\right|^{3}}+\frac{m M G\left(\boldsymbol{u}_{2 k}-\boldsymbol{u}_{1 k+1}\right)}{\left|\boldsymbol{u}_{2 k}-\boldsymbol{u}_{1 k+1}\right|^{3}}=0 \\
& \text { b) } M \ddot{\boldsymbol{u}}_{\mu k}+\frac{m M G\left(\boldsymbol{u}_{2 k}-\boldsymbol{u}_{1 k-1}\right)}{\left|\boldsymbol{u}_{2 k}-\boldsymbol{u}_{1 k-1}\right|^{3}}+\frac{m M G\left(\boldsymbol{u}_{1 k}-\boldsymbol{u}_{2 k+2}\right)}{\left|\boldsymbol{u}_{1 k}-\boldsymbol{u}_{2 k+2}\right|^{3}}=0
\end{aligned}
$$

By substituting the equations of motion of Equation (5) into Equation (4) one obtains:

$$
\text { a) } \frac{\mathrm{d} H}{\mathrm{~d} t_{k}}=0 \text { and thus } \quad H=\text { Constant }
$$

The second result we are seeking is given above by Equation (6). Since this is a conservative system, the Hamiltonian is equal to the energy. The time derivative of the Hamiltonian is equal to zero. Therefore the Hamiltonian is a constant, and thus, the energy, too, is a constant.

\section{Conclusion}

It is shown here that the very successful Kepler Newtonian model, and thus the mathematical model analyzing the effect of the gravitational propagation delay is valid. This occurs in the case when the wavelengths of the gravitational waves excited by the motion of the masses which are much larger than the system of masses. For this range of system parameters, the extension of the Newtonian Gravitational potential that includes the gravitational propagation delay is also valid. It was questioned if the solution reached in the paper "The Contribution of the Gravitational Propagation Delay to Orbital and Center of Mass Motions" [1] conserved energy? This is a conservative system and therefore, the Ha- miltonian is equal to the energy. Here, the Hamiltonian sum has been calculated from the Lagrangian sum. It is shown that the time derivative of the Hamiltonian sum is equal to zero. This implies that the Hamiltonian sum is a constant. Therefore, the energy is constant and is conserved.

\section{Acknowledgements}

I thank my wife Marlene for our discussions about the subject matter of this paper, making the text more understandable for the reader. I also thank her for proof reading, for correcting my mistakes and for her assistance in formulating 
this paper.

\section{References}

[1] Kornreich, P. (2016) The Contribution of the Gravitational Propagation Delay to Orbital and Center of Mass Motions. Journal of Modern Physics, 7, 1909-1932. https://doi.org/10.4236/jmp.2016.714169

[2] Einstein, A., Lorentz, H.A., Weyl, H. and Minkowski, H. (1952) The Principles of Relativity. Dover Publishing, Minoeola.

[3] Abbott, B.P., et al. (2016) Observation of Gravitational Waves from a Binary Black Hole Merger. Physical Review Letters, 116, Article ID: 061102.

[4] Goldstein, H. (1980) Classical Mechanics. Addison-Wesley Publishing Company, Reading MA.

[5] Dirac, P.A.M. (1938) Classical Theory of Radiating Electrons. Proceedings of the Royal Society (London A), 167, 148-169. https://doi.org/10.1098/rspa.1938.0124

[6] Kornreich, P. (2017) Reaction Mechanics for Point Objects. Journal of Applied Mathematical Physics, 4, 1909-1932.

[7] Callen, H. (1962) Thermodynamics. Wiley Co., Weinheim.

[8] Shen, S.W. (1995) Dynamics in a Discrete Nagumo Equation: Spatial Topological Chaos. SIAM Journal of Applied Mathematics, 55, 1764-1781.

https://doi.org/10.1137/S0036139994261757 


\section{Appendix A}

To include the effect of both finite and infinitesimal time increments in the derivation of the equation of motion, a sum $S$ of Lagrangians $L_{k}$ is used. The method used here is similar to the discrete Nagumo equation [8]

$$
S=\sum_{k=-\infty}^{k=\infty} L_{k}
$$

The equations of motion are obtained by inserting the sum of Lagrangians into an equation similar to the Euler Lagrange equations of motion for the Moon and the Planet.
a) $\frac{\mathrm{d}}{\mathrm{d} t_{k}} \frac{\partial S}{\partial \dot{x}_{\mu k}}-\frac{\partial S}{\partial x_{\mu k}}=0$
b) $\frac{\mathrm{d}}{\mathrm{d} t_{k}} \frac{\partial S}{\partial \dot{y}_{\mu k}}-\frac{\partial S}{\partial y_{\mu k}}=0$

This equation of motion was derived in my paper titled "Reaction Mechanics for Point Objects" [6]. By using the Lagrangians $L_{k}$ of Equation (3) in the sum of Lagrangians $S$ in Equation (A1), and substituting this sum into Equations (A2) one obtains the following discrete Nagumo [8] like equation of motion for the Moon and Planet respectively:

a) $m \ddot{x}_{\mu k}+\frac{m M G\left(x_{\mu k}-y_{\mu k-2}\right)}{2\left[\left(x_{v k}-y_{v k-2}\right)\left(x_{v k}-y_{v k-2}\right)\right]^{\frac{3}{2}}}+\frac{m M G\left(x_{\mu k}-y_{\mu k+2}\right)}{2\left[\left(x_{v k}-y_{v k+2}\right)\left(x_{v k}-y_{v k+2}\right)\right]^{\frac{3}{2}}}=0$

b) $M \ddot{y}_{\mu k}+\frac{m M G\left(y_{\mu k}-x_{\mu k-1}\right)}{2\left[\left(y_{v k}-x_{v k-1}\right)\left(y_{v k}-x_{v k-1}\right)\right]^{\frac{3}{2}}}+\frac{m M G\left(y_{\mu k}-x_{\mu k+1}\right)}{2\left[\left(y_{v k}-x_{v k+1}\right)\left(y_{v k}-x_{v k+1}\right)\right]^{\frac{3}{2}}}=0$

In order to calculate the Hamiltonian [4] of this system, it is necessary to first calculate the components of the Reaction Mechanics momentums [6] $p_{\mu k}$ and $q_{\mu k}$ of the Moon and the Planet at time step $t_{k}$
a) $p_{\mu k}=\frac{\partial\left(L_{k}+L_{k+1}\right)}{\partial \dot{x}_{\mu k}}$
b) $q_{\mu k}=\frac{\partial\left(L_{k}+L_{k+1}\right)}{\partial \dot{y}_{\mu k}}$

Only the current $L_{k}$ and the future $L_{k+1}$ Lagrangians are functions of the current coordinate components $x_{\mu k}$ and $y_{\mu k}$ of the Moon and Planet. By substituting Equation (3) for the Lagrangians $L_{k}$ and $L_{k+1}$ into equation A4 one obtains for the components of the Reaction Mechanics momentums.
a) $p_{\mu k}=m \dot{x}_{\mu k}$
b) $q_{\mu k}=M \dot{y}_{\mu k}$

The Hamiltonian sum $H$ is equal to the Lagender transform [7] of the sum of Lagrangians $S$ with respect to the velocity components.

$$
\begin{aligned}
H= & \sum_{k=-\infty}^{k=\infty}\left(p_{\mu k} \dot{x}_{\mu k}+q_{\mu k} \dot{y}_{\mu k}\right)-\sum_{k=-\infty}^{k=\infty}\left(\frac{1}{2} m \dot{x}_{\mu k} \dot{x}_{\mu k}+\frac{1}{2} M \dot{y}_{\mu k} \dot{y}_{\mu k}\right) \\
& -\sum_{k=-\infty}^{k=\infty}\left[\frac{m M G}{2 \sqrt{\left(x_{\mu k}-y_{\mu k-2}\right)\left(x_{\mu k}-y_{\mu k-2}\right)}}+\frac{m M G}{2 \sqrt{\left(y_{\mu k}-x_{\mu k-1}\right)\left(y_{\mu k}-x_{\mu k-1}\right)}}\right] \\
& -\sum_{k=-\infty}^{k=\infty}\left[\frac{m M G}{2 \sqrt{\left(x_{\mu k+2}-y_{\mu k}\right)\left(x_{\mu k+2}-y_{\mu k}\right)}}+\frac{m M G}{2 \sqrt{\left(y_{\mu k+1}-x_{\mu k}\right)\left(y_{\mu k+1}-x_{\mu k}\right)}}\right]
\end{aligned}
$$


Using Equations (A5) in Equation (A6) and collecting terms in the resulting expression.

$$
\begin{aligned}
H= & \sum_{k=-\infty}^{k=\infty}\left[\frac{p_{\mu k} p_{\mu k}}{2 m}+\frac{q_{\mu k} q_{\mu k}}{2 M}-\frac{m M G}{2 \sqrt{\left(x_{\mu k}-y_{\mu k-2}\right)\left(x_{\mu k}-y_{\mu k-2}\right)}}\right. \\
& -\frac{m M G}{2 \sqrt{\left(y_{\mu k}-x_{\mu k-1}\right)\left(y_{\mu k}-x_{\mu k-1}\right)}}-\frac{m M G}{2 \sqrt{\left(x_{\mu k+2}-y_{\mu k}\right)\left(x_{\mu k+2}-y_{\mu k}\right)}} \\
& \left.-\frac{m M G}{2 \sqrt{\left(y_{\mu k+1}-x_{\mu k}\right)\left(y_{\mu k+1}-x_{\mu k}\right)}}\right]
\end{aligned}
$$

Taking the time derivative with respect to a current time tk of the Hamiltonian sum in Equation (A7).

$$
\begin{aligned}
\frac{\mathrm{d} H}{\mathrm{~d} t_{k}}=m \dot{x}_{\mu k} \ddot{x}_{\mu k}+\frac{m M G\left(x_{\mu k}-y_{\mu k-2}\right) \dot{x}_{\mu k}}{\left[\left(x_{\mu k}-y_{\mu k-2}\right)\left(x_{\mu k}-y_{\mu k-2}\right)\right]^{\frac{3}{2}}}+\frac{m M G\left(x_{\mu k}-y_{\mu k+1}\right) \dot{x}_{\mu k}}{\left[\left(y_{\mu k+1}-x_{\mu k}\right)\left(y_{\mu k+1}-x_{\mu k}\right)\right]^{\frac{3}{2}}} \\
+M \dot{y}_{\mu k} \ddot{y}_{\mu k}+\frac{m M G\left(y_{\mu k}-x_{\mu k-1}\right) \dot{y}_{\mu k}}{\left[\left(y_{\mu k}-x_{\mu k-1}\right)\left(y_{\mu k}-x_{\mu k-1}\right)\right]^{\frac{3}{2}}}+\frac{m M G\left(y_{\mu k}-x_{\mu k+2}\right) \dot{y}_{\mu k}}{\left[\left(x_{\mu k+2}-y_{\mu k}\right)\left(x_{\mu k+2}-y_{\mu k}\right)\right]^{\frac{3}{2}}}
\end{aligned}
$$

Equations (A8) and (A3) are rewritten as Equations (4) and (5) in the main text. By substituting the equations of motion, Equations (A3) into Equation (A8) one obtains:

$$
\text { a) } \frac{\mathrm{d} H}{\mathrm{~d} t_{k}}=0 \text { and thus } H=\text { Constant }
$$

Since this is a conservative system, the Hamiltonian is equal to the energy. The time derivative of the Hamiltonian is equal to zero. Therefore, the Hamiltonian is a constant, and the energy, also is a constant. 


\section{Appendix B}

Returning to Equation (A7) to investigate the relationship between the wavelength of the gravitational wave generated by the motion of the masses $m$ and $M$, and the size of the system. It is assumed here that the wavelength of the gravitational wave is very much larger than the size of the system. In this limit the General Relativity Theory reverts to the Classical Mechanics model of nature. Therefore, one can use the much simpler Classical Mechanics for the calculations. For simplicity, neglecting the propagation delay and using the more compact vector notation one obtains for the equations of motions of the Moon and Earth from Equation (A7):

$$
\begin{aligned}
& \text { a) } \ddot{\boldsymbol{u}}_{1}+\frac{M G\left(\boldsymbol{u}_{1}-\boldsymbol{u}_{2}\right)}{\left(u_{1}^{2}-2 u_{1} u_{1}+u_{1}^{2}\right)^{\frac{3}{2}}}=0 \\
& \text { b) } \ddot{u}-\frac{m G\left(\boldsymbol{u}_{1}-\boldsymbol{u}_{2}\right)}{\left(u_{1}^{2}-2 u_{1} u_{1}+u_{1}^{2}\right)^{\frac{3}{2}}}=0
\end{aligned}
$$

It is conventional to make a transformation of variables at this point:

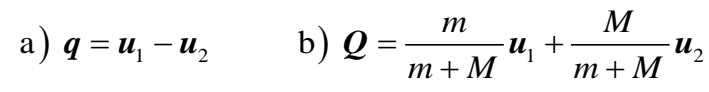

where $\boldsymbol{q}$ is the vectorial distance between objects and $Q$ is the center of mass coordinate vector. Inverting Equations (B2).

$$
\begin{array}{ll}
\text { a) } \boldsymbol{u}_{1}=\boldsymbol{Q}+\frac{M}{m+m} \boldsymbol{q} & \text { b) } \boldsymbol{u}_{2}=\boldsymbol{Q}-\frac{m}{m+M} \boldsymbol{q}
\end{array}
$$

Substituting equations B3a and B3b into Equations (B1).

$$
\begin{array}{ll}
\text { a) } \ddot{\boldsymbol{Q}}+\frac{M}{m+m} \ddot{\boldsymbol{q}}+\frac{M G \boldsymbol{q}}{q^{3}}=0 & \text { b) } \ddot{\boldsymbol{Q}}-\frac{m}{m+M} \ddot{\boldsymbol{q}}-\frac{m G \boldsymbol{q}}{q^{3}}=0
\end{array}
$$

First, subtract Equation (B4b) from Equation (B4a) and dot multiply the result by the vector $\boldsymbol{q}$. Next, subtract Equation (B4b) from Equation (B4a) and cross multiply the result by the vector $\boldsymbol{q}$. Last, multiply Equation (B4a) by $\mathrm{m}$ and equation $\mathrm{B} 4 \mathrm{~b}$ by $\mathrm{M}$ and add the resulting expressions.

$$
\begin{array}{lll}
\text { a) } \boldsymbol{q} \cdot \ddot{\boldsymbol{q}}+\frac{(m+M) G}{q}=0 & \text { b) } \boldsymbol{q} \times \ddot{\boldsymbol{q}}=0 & \text { c) }(m+M) \ddot{\boldsymbol{Q}}=0
\end{array}
$$

Equation B5c implies that the center of mass velocity $\dot{Q}$ is a constant of the motion. Next, making a transformation to cylindrical coordinates.

$$
\begin{array}{ll}
\text { a) } q_{1}=\rho \cos \theta & \text { b) } q_{2}=\rho \sin \theta
\end{array}
$$

Substituting equations B6 into Equations (B5a) and (B5b).
a) $\ddot{\rho}-\rho \dot{\theta}^{2}+\frac{(m+M) G}{\rho^{2}}=0$
b) $\hat{\boldsymbol{a}}_{3}\left(2 \rho \dot{\rho} \dot{\theta}+\rho^{2} \ddot{\theta}\right)=0$
c) $\frac{\mathrm{d}}{\mathrm{d} t} \rho^{2} \dot{\theta}=0$
d) $\rho^{2} \dot{\theta}=\ell$
e) $\dot{\theta}=\frac{\ell}{\rho^{2}}$

Here $\lambda$ is the angular momentum which is a constant of the motion. By substituting Equation (B7e) into Equation (B7a) one obtains the equation of motion. 


$$
\frac{\mathrm{d}^{2} \rho}{\mathrm{d} t^{2}}-\frac{\ell^{2}}{\rho^{3}}+\frac{(m+M) G}{\rho^{2}}=0
$$

where $\rho$ is the distance between objects. Approximating the distance between objects $\rho$ by its average value $\mathrm{R}$ and a small oscillating portion $\mathrm{rcos} \omega \mathrm{t}$.

$$
\rho=R+r \cos \omega t
$$

The oscillation of the distance between objects is observed as an eccentric orbit where the distance between objects change periodically. Substituting Equation (B9) for the distance between objects $\rho$ into Equation (B8) and expanding the resulting expression to first order in the small parameter $\frac{r}{R} \cos \omega t$.

$$
-\omega^{2} r \cos \omega t-\frac{\ell^{2}}{R^{3}}+\frac{3 \ell^{2}}{R^{4}} r \cos \omega t+\frac{(m+M) G}{R^{2}}-\frac{2(m+M) G}{R^{3}} r \cos \omega t \approx 0
$$

Collecting terms in Equation (B10):

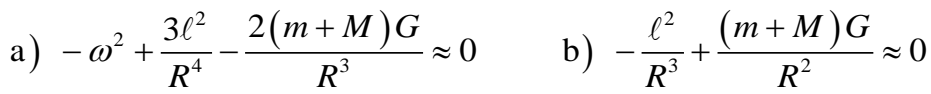

Solving Equation (b11b) for the angular momentum $\lambda$ and substituting the result into Equation (B11a).

$$
\omega^{2}=\frac{(m+M) G}{R^{3}}
$$

Solving this equation for the oscillating frequency $f$.

$$
\text { a) } f=\frac{1}{2 \pi R} \sqrt{\frac{(m+M) G}{R}} \quad \text { where } \quad \text { b) } \omega=2 \pi f
$$

The oscillating and orbiting objects will cause gravitational waves in the space-time continuum surrounding the objects, see Figure 1. The gravitational waves travel with the speed of light. The wavelength $\lambda$ of this wave is equal to:

$$
\begin{array}{ll}
\text { a) } \lambda=\frac{c}{f} & \text { b) } \lambda=2 \pi R \sqrt{\frac{2 R}{r_{s s}}}
\end{array}
$$

where $R$ is the average distance between objects. Here $r_{s s}$ is the Schwarzschild radius of the sum mass given by:

$$
r_{s s}=\frac{2(m+M) G}{c^{2}}
$$

Equations (B14b) and (B15) are the results we are seeking. Equations (B14b) and (B15) are rewritten as Equations (1) and (2) in the main text. 
Submit or recommend next manuscript to SCIRP and we will provide best service for you:

Accepting pre-submission inquiries through Email, Facebook, LinkedIn, Twitter, etc. A wide selection of journals (inclusive of 9 subjects, more than 200 journals)

Providing 24-hour high-quality service

User-friendly online submission system

Fair and swift peer-review system

Efficient typesetting and proofreading procedure

Display of the result of downloads and visits, as well as the number of cited articles Maximum dissemination of your research work

Submit your manuscript at: http://papersubmission.scirp.org/

Or contact jamp@scirp.org 